L'heuristique de la littérature grise sur le développement participatif du Bassin congolais

Daou V. Joiris et Patrice Bigombe Logo

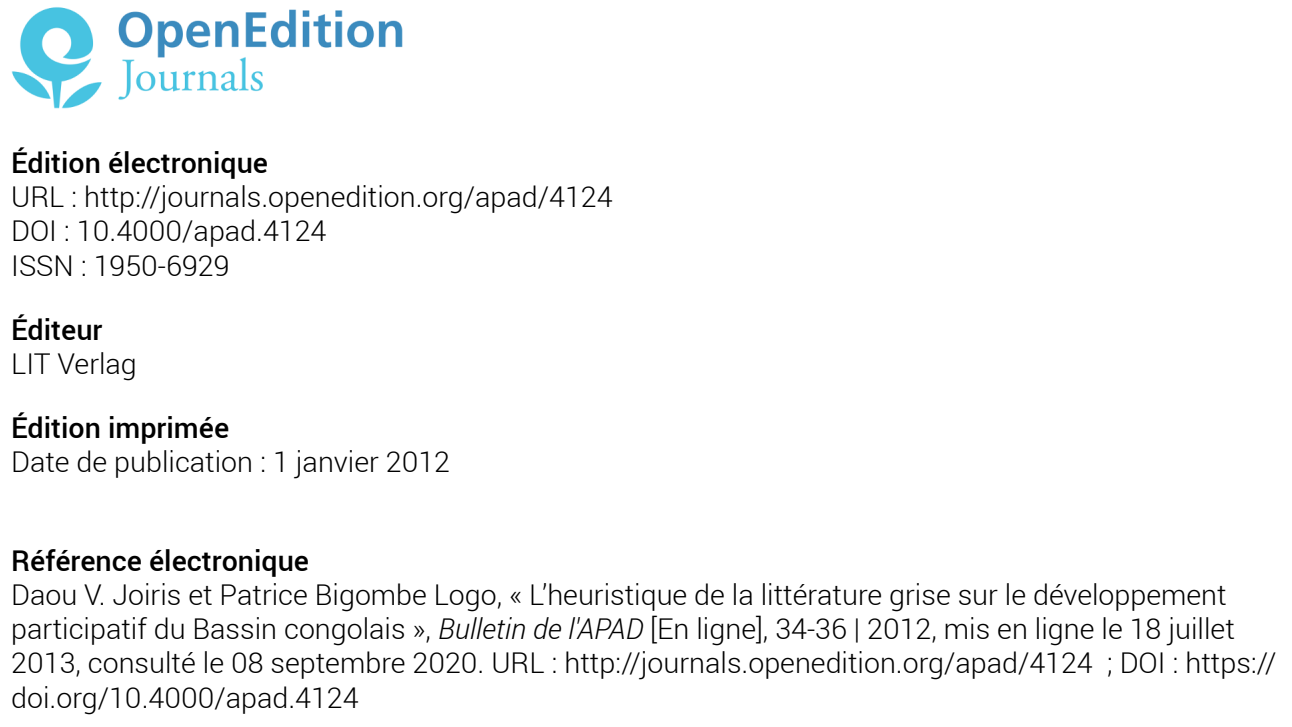

Ce document a été généré automatiquement le 8 septembre 2020.

Bulletin de l'APAD 


\title{
L'heuristique de la littérature grise sur le développement participatif du Bassin congolais
}

\author{
Daou V. Joiris et Patrice Bigombe Logo
}

\section{Introduction}

1 On a déjà souligné dans la littérature socio-anthropologique du développement, le problème d'un « chaînon manquant » et de difficultés à communiquer des résultats opérationnels aux décideurs (Olivier de Sardan, 2004 ; Lavigne Delville, 2007 ; Bako Arifari, 2007 ; Le Meur, 2007). Comme le rappelle bien Philippe Lavigne Delville, « il n'y a pas un chaînon manquant, mais des chaînons manquants, de nature différente. D'une part, des chaînons manquants cognitifs, qui permettent de faire les liens de raisonnement entre une lecture socio-anthropologique des dynamiques sociales et les objets d'action des développeurs, en construisant à la fois les raisonnements intermédiaires [...] ceux qui permettent [par exemple] de passer des pouvoirs locaux aux modes de validation politique des projets [...] produisant ainsi de nouveaux référentiels structurant l'action [...], et les objets d'interface qui font partie de l'univers de pensée et d'action des développeurs [...] mais qui doivent être définis et pensés en termes à la fois sociologiquement réalistes [...] et suffisamment opérationnels pour que les développeurs puissent situer leur action à partir d'eux et ainsi ancrer leur pratique [...]. D'autre part, des chaînons manquants en termes institutionnels, des marginaux sécants qu'ils soient situés dans l'espace de la recherche ou dans celui de l'action, qui maîtrisent suffisamment les deux univers de sens, leurs règles internes, leurs référentiels propres, pour pouvoir jouer le rôle de traducteur d'un univers de sens à un autre [...]. » (Lavigne Delville, 2007: 147).

2 Notre propos concerne un aspect en amont du « chaînon manquant cognitif » et correspond à une tentative d'explication de la difficulté vécue par les sciences sociales à établir des «liens de raisonnement entre une lecture socio- 
anthropologique des dynamiques sociales et les objets d'action des développeurs " (op. cit.). Nous nous focalisons sur un versant particulier de l'activité socioanthropologique, à savoir la littérature grise, celle des rapports de consultance et d'expertises, qui est produite, - pour reprendre l'expression de Bennett (1996) - en dehors de l'institution anthropologique, au sein de l'institution projet. Le terme "socio-anthropologique" est employé ici dans un sens très large, englobant l'ensemble des professions qui se prêtent à des «expertises » et "consultances » dites " socio-économiques » (sociologues et anthropologues, mais aussi agronomes, spécialistes du développement rural, économistes, politologues, psychologues, etc.). C'est donc sur la production de ce type de savoir, appliqué dans le cadre des " expertises", des "consultances ", particulièrement in fine, la formulation de termes de référence conformément au cadre logique et de recommandations adressées aux décideurs que porte notre contribution.

3 Nous partons de l'idée que le contexte d'exercice de la démarche appliquée au sein $\mathrm{du}$ dispositif projet est déterminant $\mathrm{du}$ point de vue cognitif et que l'historicité, la temporalité et la sectorialité de ce dernier détermine la portée heuristique des connaissances socio-anthropologiques. La production de données au sein d'un projet de développement, même si celle-ci résulte en partie d'un recours à la littérature scientifique, comporte, selon nous, des spécificités cognitives propres au champ du développement et à la façon dont les ressortissants des sciences sociales s'adaptent à un certain modus vivendi vis-à-vis des décideurs, des gestionnaires de projets et d'autres praticiens du développement. C'est un détail, pour certains ; et pour d'autres, un rappel. Cela peut paraître trivial. Selon nous, ce type de situation requiert toute son importance dans la mesure où ce formatage cognitif déterminerait la nature des recommandations formulées par nos disciplines, et bien entendu la portée plus ou moins réaliste, opérationnelle, de celles-ci.

4 La typologie adoptée pour mener à bien cette réflexion repose sur la distinction essentielle entre des savoirs produits au sein de l'institution projet et de l'institution académique, que ceux-ci soient de nature fondamentale (au sens de recherche fondamentale) ou appliquée. Ainsi, l'engagement en socio-anthropologie (activiste, politique, populiste, etc.) constitue une catégorie transversale à l'ensemble de ces recherches, l'engagement étant toutefois généralement plus marqué du côté académique (fondamental et appliqué) que du côté projet. La limite entre ces deux types de savoirs, de consultance et académique, peut paraître ténue en raison de nombreux recouvrements. Pourtant, il ne s'agit pas du même dispositif cognitif. Les termes de référence (TDR) d'une expertise ne s'élaborent pas de la même manière que le protocole d'une recherche académique. Les cartes mentales mobilisées dans l'une ou l'autre de ces configurations (TDR, recherche académique) ne sont pas du même ordre. C'est ce que nous souhaitons montrer sur la base d'un cas concret.

5 Notre démonstration s'appuie sur la comparaison entre la littérature grise et la production académique relatives aux projets de développement à vocation participative $^{1}$ au sein des politiques de gestion durable des forêts du Bassin congolais, depuis les années 1980 . Du point de vue méthodologique, l'analyse proposée ne résulte pas d'une ethnographie systématique de ladite littérature grise. Il s'agit à ce stade d'une démonstration intuitive, élaborée a posteriori, sur 
base d'une bonne connaissance des rapports de consultance et de la mise en perspective des recommandations issues de ces derniers par rapport à l'étude des enjeux locaux suscités par ces politiques. ${ }^{2}$

\section{Le terreau projet des clivages cognitifs}

6 Ce que produit comme connaissances la littérature grise en sciences sociales est directement dépendant des conditions de son exercice, c'est-à-dire du cadre cognitif de l'approche projet. La pratique projet, y compris les études et les interventions commanditées par ces derniers aux sciences sociales, s'exerce verticalement et horizontalement. Verticalement, dans le sens où elle s'inscrit dans une temporalité particulière, essentiellement dans le présent, en rupture avec le passé, et dans une temporalité cyclique. Horizontalement, dans le sens où la pratique projet est sectorielle, et de ce fait cloisonnée.

7 Dans les pages qui suivent, nous proposons de montrer comment ces trois aspects de la pratique projet contribuent à la production de connaissances à la fois a-historiques, sectorielles et non cumulatives, et comment ces aspects mènent à un cadre d'analyse limité à des interprétations non comparatives et non prédictives (voir tableau1).

8 Tableau 1 : Profil cognitif des sciences sociales au sein des institutions projet et académiques

\begin{tabular}{|l|l|}
\hline $\begin{array}{l}\text { CONNAISSANCES PRODUITES AU SEIN } \\
\text { DE L'INSTITUTION PROJET } \\
\text { (RECHERCHE APPLIQUEE) }\end{array}$ & $\begin{array}{l}\text { CONNAISSANCES PRODUITES AU SEIN DE } \\
\text { L'INSTITUTION ACADEMIQUE } \\
\text { (RECHERCHE FONDAMENTALE ET } \\
\text { APPLIQUEE) }\end{array}$ \\
\hline A-historiques & Historiques \\
\hline Sectorielles & Plurisectorielles \\
\hline Non cumulatives & Cumulatives \\
\hline Non comparatives & Comparatives \\
\hline Non prédictives & Non prédictives \\
\hline
\end{tabular}

\section{Des connaissances a-historiques, sectorielles et non cumulatives}

\section{Des connaissances a-historiques}

9 Dans une contribution récente intitulée "Anthropology, Development and the 'Perpetual Present'. Knowledge, Power and Practice» (2009), Lewis rappelle les nombreuses réflexions préalables sur la temporalité particulière des projets. L'anthropologie du développement, écrit-il (op. cit. : 53-54), notamment au travers 
d'études de cas et de monographies très fines, montre la tendance a-historique qu'ont les agences de développement à vivre dans le "perpétuel présent ", à reformuler leurs approches et à se projeter dans le futur plutôt que de se penser en continuité d'événements passés. Il cite, entre autres, les recherches sur la production cotonnière au Mozambique d'Isaacman (1997) qui parle de "l'amnésie historique " des projets, les cadres du développement étant focalisés sur des projections dans le futur plutôt que sur une compréhension des logiques économico-politiques héritées des contextes coloniaux.

10 Rien de neuf à l'horizon. A la suite d'autres auteurs qui tenaient des propos similaires, Olivier de Sardan insistait en 1995 sur le fait que «[...] tout projet intervient [...] dans un milieu qui a déjà subi de nombreuses interventions précédentes et qui en garde la trace, bien que 'la tendance naturelle dans un projet (soit) toujours de considérer que l'histoire démarre avec le projet, de sous-estimer systématiquement tout ce qui s'est fait auparavant et de surestimer l'impact du projet' » (op. cit : 127, citant Gentil et Dufumier, $1984: 25$ ). La « tendance naturelle » des projets à s'inscrire dans une temporalité a-historique est donc connue. En revanche, l'influence de cette a-historicité sur la production de connaissances de consultance semble passée sous silence.

11 La pratique projet s'inscrit dans une discontinuité historique d'un double point de vue: de celui de l'histoire des populations concernées par son intervention, et de sa propre histoire en tant que projet. L'évocation du passé des communautés concernées par l'intervention des projets est souvent absente des termes de référence (TDR) et des commandes d'études adressées aux sciences sociales. Cela signifie en d'autres termes que la conception et la mise en œuvre des projets se réalisent en faisant abstraction des événements historiques qu'ont vécu les populations ciblées ou concernées par ces derniers. Par ailleurs, la pratique projet est marquée par un autre mode d'a-historicité. En effet, celle-ci s'inscrit dans une discontinuité par rapport à sa propre histoire ou à l'histoire d'autres projets ayant une expérience du même ordre. Elle est plutôt caractérisée par des préoccupations de l'ordre de la projection dans l'avenir et par une tendance omniprésente à la confusion entre les intentions (le futur) et les réalisations (le présent).

12 Dans le cas des politiques participatives du Bassin congolais, le contexte diachronique des milieux sociaux concernés par l'intervention de projet est largement occulté. Dans les TDR, la rubrique "présentation du contexte d'intervention ", l'histoire localisée des populations ciblées, précoloniale, coloniale et contemporaine, est généralement très faiblement, voire pas du tout, documentée. De même, dans la rubrique relative aux thématiques d'enquête commanditées aux sciences sociales, un accroissement des connaissances sur l'histoire localisée est loin de représenter une priorité. Les moyens financiers n'y sont pas consacrés, que ce soit pour réaliser une recherche bibliographique ou des enquêtes de terrain.

13 Le même constat s'impose concernant une tendance à une forme d' "amnésie historique » quant à l'intervention même des projets. Le suivi actif des deux premières phases du programme régional ECOFAC ainsi qu'une documentation sur les phases suivantes et sur des projets complémentaires, montrent clairement, sur une quinzaine d'années, à quel point l'appropriation des données en sciences sociales est superficielle et répétitive (Joiris, 2000a, 2000b, 1997, 1996; Joiris et 
Binot, 2001). De phase en phase, l'histoire pourtant sérieusement mouvementée des interactions entre le programme et les riverains des aires protégées est pratiquement absente. A chaque fois, les TDR reprennent, à peu de choses près, les mêmes informations dans les mêmes rubriques comme si le projet amorçait un premier contact dans un terrain vierge. L'histoire des interactions entre le projet et les populations n'est pas mobilisée par les concepteurs et les gestionnaires des projets en tant que caractéristique du contexte d'intervention.

\section{Des connaissances sectorielles}

14 La pratique projet s'exerçant de façon sectorielle et séparée en fonction des sources de financement, l'expertise en sciences sociales réalisée dans ce contexte débouche sur une production sectorielle et morcelée des connaissances.

15 Dans le domaine participatif, à l'échelle du Bassin congolais, la pratique professionnelle des sciences sociales spécialisées sur ces aspects est fortement clivée. En rapport avec le secteur de l'exploitation industrielle du bois, des chercheurs en sciences humaines sont spécialisés sur les questions de redistribution locale de la fiscalité forestière décentralisée (dont la fameuse Rente Forestière Annuelle au Cameroun) et de forêts communautaires. Les zones de chasse à vocation communautaire correspondent à une autre spécialisation, tandis que les aires protégées en constituent une troisième. Pour présenter cette partition avec plus de souplesse, disons qu'en dépit du fait que le consultant en sciences sociales réalise de facto des appuis aux différents secteurs, la littérature grise correspondante couvre ces secteurs indépendamment les uns des autres.

16 La pratique professionnelle des sciences sociales spécialisées sur ces aspects est d'autant plus clivée qu'elle l'est également en fonction des sources de financement. Les rapports produits pour le secteur privé, l'Etat, à la demande des communautés, pour un bailleur de fonds ou une ONG sont avant tout la propriété du commanditaire, avec des degrés plus ou moins importants de confidentialité. Il s'agit donc d'une production de connaissances au sein de sphères séparées dont les conclusions ne circulent pas aisément. Nous reviendrons sur ce point important, car il s'agit bien entendu, au même titre que de l'« amnésie historique » soulignée plus haut, d'une raison majeure à la difficulté de dégager des recommandations opérationnelles.

\section{Des connaissances non cumulatives, répétitives}

17 L'épistémologie des logiques sous-jacentes à la gestion des projets montre clairement que celle-ci présente un caractère cyclique avec une tendance forte à la reproduction de prémisses récurrentes (évolutionnistes, occidentalo-centristes, néo-malthusiens, etc.). L'exercice, dans ce contexte de la production de connaissances en sciences sociales, indique également une tendance à la mobilisation de données non cumulatives. Des analyses antérieures relatives aux interactions projets-population ne sont pas convoquées. Cette absence de capitalisation des expériences se manifeste verticalement, en raison d'une propension à l'a-historicité et horizontalement, en raison du cloisonnement sectoriel des interventions, mais surtout en raison d'une faible habitude de la part 
des projets à valoriser l'évolution d'une intervention donnée et à considérer que cette information puisse contribuer à l'affinement d'un protocole.

18 La réalisation de consultances liées à la mise en œuvre des politiques participatives dans le Bassin congolais est indiscutablement caractérisée par une absence de recours aux expériences précédentes. Le cas du dysfonctionnement chronique, depuis les années 1980, des "comités de gestion", quel que soit le secteur concerné, en est un exemple parlant. Des études en anthropologie du développement et en politologie montrent que les dysfonctionnements de ces comités sont récurrents. Les difficultés rencontrées seraient dues au caractère " exogène » des comités ${ }^{3}$. Malgré ce constat, et malgré les moyens considérables mis en œuvre pour promouvoir divers appuis technicistes (méthodes de résolution des conflits, enquêtes rapides, etc.), les comités de gestion continuent jusqu'à présent d'être conçus d'après les mêmes principes exogènes. Et les sciences sociales, de leur côté, continuent de formuler les mêmes critiques à ce propos... Sur ce point, mais il ne s'agit que d'un exemple parmi d'autres, les praticiens en sciences sociales ne s'appuient pas sur l'état des connaissances qui ressort de la littérature académique pour fournir les compréhensions les plus appropriées. Ils se réfèrent à l'état des connaissances de la littérature grise et ce n'est qu'à partir de là qu'ils fournissent des recommandations. Celles-ci sont répétitives puisque les données mobilisées sont non cumulatives.

\section{Un cadre d'analyse dominé par l'absence de comparatisme et de prédictibilité}

\section{Des interprétations non comparatives}

19 Les vertus heuristiques du comparatisme ne sont plus à démontrer. Il s'agit là d'un des bastions méthodologiques de l'anthropologie et de la démarche scientifique en général. «[...] Penser comparativement permet de se défaire d'une simple collection de faits et de poser les questions opératoires, celles qui naissent de la comparaison, de la mise en perspective, du croisement de configurations semblables mais autrement agencées, ou alors radicalement différentes. Il n'y a pas de comparatisme expérimental qui n'oblige à penser autrement ce qui nous semble faire partie spontanément du sens commun et des catégories familières.» (Detienne, 2009 : 137).

20 Or, le caractère sectoriel et morcelé de la littérature grise débouche sur un patrimoine de connaissances qui ne se prête pas aisément au comparatisme. Des données a-historiques, sectorielles et non-cumulatives peuvent difficilement être soumises à des analyses comparatistes et donc déboucher sur ce que Detienne (op. cit.) qualifie de « questions opératoires».

21 Concernant les politiques participatives dans le Bassin congolais, la comparaison des effets des approches développementistes, tous secteurs et pays confondus, donc dans une perspective transsectorielle et transrégionale, fournit des résultats surprenants auxquels les approches cloisonnées de la consultance habituelle n'aboutissent pas, tout au moins de façon aussi évidente. Cette mise en perspective montre très clairement que l'essentiel des difficultés rencontrées sur le terrain, à 
l'instar du dysfonctionnement chronique des comités de gestion mentionné plus haut, est imputable tant au contexte socio-politique et néocolonial d'implantation qu'au modèle de développement lui-même (Joiris et Bigombe, 2010b). Il ressort également de l'analyse académique comparative, quel que soit le secteur et le pays d'intervention, que la «Policy-pratique " participative résulte d'un formatage du même ordre, produit en réaction à des logiques et stratégies d'acteurs également du même ordre, autours de trois principaux enjeux récurrents : l'accès à la terre et à ses ressources, l'accès aux ressources financières, en nature et symbolique, les représentativités, s'exerçant dans des milieux où règnent des rapports de domination et de clientélisme.

\section{Des interprétations non prédictives}

De la sorte, la pratique-projet, y compris les études et les interventions commanditées aux sciences sociales, s'exerce abstraction faite des connaissances acquises sur les enjeux récurrents suscités par les politiques de développement, donc sur ses modalités d'appropriation. Cette approche qui consiste à s'inscrire dans une discontinuité d'expériences enferme la pratique-projet, et celle des sciences sociales dont les études et les interventions se réalisent en leur sein, dans une vision du futur limitée aux objectifs de projet et aux logiques supposées leur être sous-jacentes du point de vue du développeur. Elle contribue à la production de connaissances technicistes ou fonctionnelles à court terme, qui manquent de recul critique et de perspective visionnaire pour envisager tous les futurs possibles.

23 Cette posture s'inscrit en porte-à-faux vis-à-vis des principes élémentaires de management. Les gestionnaires des politiques publiques s'accordent pour dire qu'" [...] un processus de décision est d'abord une forme de négociation et d'arrangement mutuel entre les acteurs, une démarche 'pas-à-pas' dans laquelle le décideur, loin de chercher à brusquer les choses, dans une sorte de logique de rupture, va entreprendre de modifier progressivement et de manière continue le système sur lequel il veut revenir. Une telle approche débouche sur un modèle d'action dans lequel le décideur, loin d'affirmer des objectifs fixés une fois pour toutes, n'hésitera pas à modifier ses buts en fonction des résistances qu'il rencontre, à faire des concessions, à multiplier les alliances, quitte à réviser ses ambitions, à jouer avec le temps, ce qui suppose d'être patient, à privilégier les procédures au détriment des objectifs et à n'envisager les solutions qu'en fonction des moyens disponibles » (Muller, $2008: 37-38$ ).

24 Le cadre logique des projets n'intègre pas, en tant que "risques", en premier lieu, le décalage entre les intentions et les réalisations observées dans la mise en place de toute politique publique. Deuxièmement, les modalités d'appropriation connues dans des contextes apparentés ne sont pas répertoriées en tant que scenarii probables. A titre d'exemple, la littérature grise sur les approches participatives mises en œuvre dans le Bassin congolais, concernant la gestion des aires protégées, n'envisage pas, dans le cadre logique, l'éventualité hautement probable de conflits ou de tensions entre le projet et les populations riveraines. Alors que l'ensemble de la littérature en anthropologie du développement sur la gestion sociale des aires protégées témoigne de l'existence intrinsèque de ces 
conflits, dans la pratique-projet, la thématique des interactions conflictuelles apparaît comme un tabou, un « impensé du développement ».

25 Dans le Bassin congolais, tout au moins concernant les approches participatives investiguées au sein de notre programme de recherche GEPAC, d'autres logiques et stratégies d'acteurs, relevées dans la littérature anthropologique du développement comme inhérentes à la mise en œuvre de certaines politiques, sont frappées d'invisibilité : tout ce qui a trait aux enjeux fonciers, aux enjeux de représentativité administrative et politicienne, aux rapports de domination, de clientélisme et de corruption. Ce dernier trait majeur dans lequel s'inscrit la pratique en sciences sociales au sein de l'institution projet entrave cette dernière dans la formulation de pronostics, de scenarii, de prédictions. La tendance des sciences sociales appliquées à produire des connaissances non prédictives est cependant renforcée par une tradition académique selon laquelle, contrairement aux sciences naturelles, la complexité des interactions sociales ne se prêterait que très difficilement à des projections dans l'avenir (Picouet et al. 2004 : 17-43).

26 Ceci dit, et quelle que soit l'ampleur effective de l'imprédictibilité en sciences sociales, la littérature grise recourt très faiblement aux grilles d'analyse produites dans le cadre académique. C'est là se priver de données pourtant disponibles dans la hotte du socio-anthropologue du développement. Dans le cas de notre étude comparative sur les approches participatives dans le Bassin congolais, la récurrence d'enjeux d'accès à la terre, aux ressources et de représentativité apparait comme une hypothèse forte qui pourrait faire office de grille d'analyse opératoire dans la contribution au cadre logique et la formulation de recommandations.

\section{La confusion entre recommandations structurelles et conjoncturelles}

Lorsqu'il parle d' " amnésie structurelle », Bierschenk (2007 : 13) évoque comment les effets combinés des interventions de développement échappent à «l'approche empirique qui tend à se focaliser sur un projet particulier » et à la difficulté à " mettre en relation données empiriques relatives à des micro-évènements - pour laquelle une anthropologie du développement centrée sur les acteurs est particulièrement bien équipée - avec des processus de plus grande envergure - qui sont plus difficiles à saisir avec l'outillage méthodologique classique de l'anthropologie.» (op. cit.). «Une grande partie de l'anthropologie du développement ", écrit-il encore, "n'a pas réussi à enchâsser ses micro-histoires dans des récits plus larges et à faire le lien entre analyse locale et tendances globales » (ibid.).

28 L'histoire de l'expertise en sciences sociales appliquée aux politiques participatives du Bassin congolais montre une incapacité à dépasser des clivages analytiques, à s'inscrire dans une pratique d'expériences cumulatives, et de ce fait à distinguer les contraintes structurelles des contraintes conjoncturelles. Par "contraintes structurelles", nous entendons ce qui se trouve au fondement historique, social, économique et politique du milieu dans lequel intervient un projet, à savoir, pour le Bassin congolais, l'absence de mouvements sociaux, les 
reconfigurations opaques en réaction aux politiques, le néocolonialisme, l'absence de démocratie, le clientélisme et la corruption.

29 Le projet CUREF, en Guinée Equatoriale, malgré le régime dictatorial sanguinaire qui y sévit depuis l'indépendance, présente paradoxalement un formatage participatif identique à celui implanté dans les régimes pluralistes des pays voisins (Joiris et Cayuela Serrano, 1997). En outre, les réseaux diasporiques nés de la déliquescence politique et économique du pays, soit aux alentours de $50 \%$ de la population masculine dans certaines régions rurales, ne sont pas intégrés au montage de projet. Dans un tel contexte, compte tenu de l'ampleur des contraintes structurelles, les recommandations des sciences sociales, pour autant qu'elles aient l'opportunité d'être exprimées, débordent largement la marge de manœuvre des gestionnaires de projet. Toujours en Guinée Equatoriale, la notion coloniale de "réservas de poblados", une forme de gestion communautaire génératrice de sécurité foncière et d'opportunités économiques avant la lettre, n'a pas été intégrée dans les nouvelles législations forestières des années 1980. Les communautés qui s'étaient appropriées des «reservas de poblados» du temps des Espagnols en ont été dépossédées sous l'ère de la participation... A nouveau, les enjeux gouvernementaux et macro-économiques sous-jacents à de telles dispositions, et leurs effets pervers sur les communautés sensées bénéficier du développement participatif, relèvent de contraintes structurelles en dehors de la sphère d'influence des projets.

30 Par " contraintes conjoncturelles ", nous faisons allusion aux conjectures très localisées auxquelles est confronté un projet de développement, ce qui pourrait donner lieu à des ajustements de projet en vue d'un meilleur cadrage avec le milieu d'intervention. Dans notre étude GEPAC sur les comités de gestion de la rente forestière annuelle (RFA), au sud Cameroun, Mbetoumou et al. (2010) montrent que la forte dispersion géographique de l'habitat est incompatible avec un bon fonctionnement des comités de gestion, et qu'afin de décentraliser le dispositifprojet de façon plus opératoire, les élites locales ont créé des sous-comités de gestion. Dans ce cas, s'inspirer des solutions innovantes que le groupe cible a mis en place pour s'approprier le modèle développementiste, et légitimer ses actions, semblent correspondre à une recommandation réaliste au regard des attributions d'un projet.

\section{Conclusion}

31 Les incompatibilités entre le contexte projet et le contexte académique dans la production de données soulignent clairement le caractère enfermant des recommandations produites dans l'exercice de la consultance en sciences sociales. Le "chaînon manquant» cognitif relatif à la formulation de recommandations opératoires et à l'effort d'intégration de mécanismes sociologiques au sein des modèles de développement, représente un enjeu majeur de légitimité de nos disciplines dans leurs spécialisations "appliquées». Le constat d'irréductibilité entre les connaissances produites au sein de l'institution projet et de l'institution académique est incontournable. C'est là un fait sans appel. Pour autant, œuvrer dans le sens de meilleurs ponts entre la recherche fondamentale /appliquée et l'univers projet, ainsi que dans le sens de compréhensions lisibles des 
appropriations locales dont fait l'objet la «Policy-pratique » devrait certainement contribuer à créer un maillage fort. C'est là tout un domaine d'expertise à développer en sciences sociales via l'enseignement, la formation des consultants et leurs expérimentations de terrain dans l'interface avec les opérateurs de développement.

\section{BIBLIOGRAPHIE}

Arnoldussen, D., Assenmaker P., Bigombe Logo P., Binot A., Cogels S., Guedje Chaungueu N., Joiris D.V., Lejoly J., Romainville M., Roulet P.-A. \& T. Trefon 2008. « Gouvernance et environnement en Afrique centrale : le modèle participatif en question. Introduction », in Roulet P.-A. et

P. Assenmaker (eds), Gouvernance et environnement en Afrique centrale : le modèle participatif en question. Tervuren : Musée Royal de l'Afrique Centrale, pp. 11-17.

Bako Arifari, N. 2007. « La médiation socio-anthropologique entre savoir et action. Plaidoyer pour un métier de médiateur en action publique ", in Bierschenk T., Blundo G., Jaffré Y. et M. Tidjani Alou (eds), Une anthropologie entre rigueur et engagement. Essais autour de l'œuvre de Jean-Pierre Olivier de Sardan, Leiden, Paris : APAD-Karthala: 175-200.

Bennett, J. W. 1996. « Applied and action anthropology: Ideological and conceptual aspects », Current anthropology 37: 23-53.

Bierschenk, T. 2009. Anthropologie et Développement: Historiciser et localiser les approches. APAD Bulletin 31-32: “Inventing and Mobilising the Local”, (ed.) S. Hagberg, 161-192.

Bigombe Logo, P., Roulet, P.-A. 2010. « Genèse et évolution des zones d'intérêt cynégétique à gestion communautaire au sud-est du Cameroun », in Joiris D.V. \& P. Bigombe Logo (eds), Gestion participative des forêts d'Afrique centrale. Un modèle à l'épreuve de la réalité. Versailles : Editions QUAE, Collection Synthèses.

Bigombe Logo, P., Yamo, A., Ngonde, B. 2010. « Les forêts communautaires au village ", in Joiris D.V. \& P. Bigombe Logo (eds), Gestion participative des forêts d'Afrique centrale. Un modèle à l'épreuve de la réalité. Versailles : Editions QUAE, Collection Synthèses.

Binot, A. \& D.V. Joiris 2007. « Règles d'accès et gestion des ressources pour les acteurs des périphéries d'aires protégées : foncier et conservation de la faune en Afrique subtropicale. » Vertigo (La revue électronique en sciences de l'environnement), Hors Série $4:$ Les frontières de la question foncière : enchâssement social des droits et politiques publiques.

Binot, A., Hanon L., Joiris D.V. Et D. Dulieu 2009. « The challenge of participatory natural resource management with mobile herders at the scale of a Sub-Saharan African protected area». Biodiversity and Conservation, vol., $18, \mathrm{n}^{\circ} 10$.

Binot, A., Hanon, L., Ndotam Tatila, I., Joiris, D.V. 2007. «L'aménagement de territoires multiusages en périphérie d'une aire protégée africaine: entre enjeux de conservation et de développement. Le cas du parc national de Zakouma (Sud-Est du Tchad) ». Gestion participative en Afrique centrale. Quatre études de cas. 
Binot, A., Joiris, D.V., Hanon, L. 2010. «L'échec de la sécurisation foncière dans les aires protégées ». in Joiris D.V. et P. Bigombe Logo (eds)., Gestion participative des forêts d'Afrique centrale. Un modèle à l'épreuve de la réalité. Versailles : Editions QUAE, Collection Synthèses.

Cooke, B., Kothari, U. 2001. Participation : The new tyranny? London : Zed Books.

Cooper, F. \& R. Packard 1997. "International development in the social sciences - Introduction. », in Cooper and Packerd (eds) International development and the social sciences. Essays on the history and politics of knowledge, Berkeley: University of California Press: 1-41.

Detienne, M. 2009. Comparer l'incomparable. Paris: Editions du Seuil (Points, Essais).

Escobar, A. 2008. Territories of difference. Place, movements, life, redes. Durham and London: Duke University Press. 435 p. ISBN: 978-0-8223-4327-1

Gentil, D. \& M. Dufumier 1984. Le suivi évaluation dans les projets de développement rural. Orientations méthodologiques, AMIRA, 44 (mult.).

Hagberg, S. \& C. Widmark (eds), 2009. The ethnographic practice and public aid. Methods and meanings in development cooperation. Uppsala Studies in Cultural Anthropology $\mathrm{N}^{\circ} 45$. Uppsala : Acta Universitatis Upsaliensis.

Igoe, J., \& D. Brockington 2007. Neoliberal Conservation: A Brief Introduction, Conservation and Society 5 (4): 432-449.

Isaacman, A. 1997. Historical amnesia or the logic of capital accumulation: cotton production in colonial and postcolonial Mozambique, Environment and Planning D: Society and Space 15: 757-790.

Joiris, D.V. \& A. Binot 2001. Synthèse régionale des expertises anthropologiques réalisées de 1997 à 1999 pour le programme ECOFAC (phase II) et recommandations pour la phase III. AGRECOCTFT, 125 p. + annexes.

Joiris, D.V. 1996. Synthèse régionale des expertises anthropologiques réalisées de 1993 à 1996 dans le cadre de la première phase du programme ECOFAC au Cameroun, Gabon, Congo et en République Centrafricaine. Rapport ECOFAC, AGRECO-CTFT.

-. 1997. «La nature des uns et la nature des autres : mythe et réalité du monde rural face aux aires protégées d'Afrique Centrale », Civilisations, vol. XLIV, n²1-2, pp. 95-103.

-. 2000a. L'expertise en sciences humaines. Rapport final APFT (UE - DG VIII), pp. 601-616.

-. 2000b. La gestion participative et le développement intégré des aires protégées. Rapport final APFT (UE - DG VIII).

Joiris, D.V., Bigombe Logo, P. 2010. «Introduction : Des politiques participatives multiformes ». In Joiris D.V. et P. Bigombe Logo (Eds). La gestion participative des forêts d'Afrique centrale. Un modèle à l'épreuve de la réalité. Versailles : Editions QUAE, Collection Synthèses.

Joiris, D.V., Bigombe Logo, P. 2010. « Conclusion : Les enjeux locaux autour d'un mode de gouvernance anti-participatif ». In Joiris D.V. \& P. Bigombe Logo (eds), La gestion participative des forêts d'Afrique centrale. Un modèle à l'épreuve de la réalité. Versailles : Editions QUAE, Collection Synthèses.

Joiris D.V. \& N. Cayuela Serrano 1997. Mise en place de la Mision etno-sociologica en Guinea Ecuatorial, Marzo 1997. Rapport CUREF, BDPA-SCETAGRI/SECA/AGRER.

Lavigne Delville, Ph. 2007. «A la recherche du chaînon manquant. Construire des articulations entre recherche en sciences sociales et pratiques du développement ». In Bierschenk T., Blundo 
G., Jaffré Y. \& M. Tidjani Alou (eds), Une anthropologie entre rigueur et engagement. Essais autour de l'œuvre de Jean-Pierre Olivier de Sardan. Leiden, Paris : APAD-Karthala. pp. 127-150.

Le Meur, P.-Y. 2007. « Anthropologie et développement. Une relation à plaisanterie?». In Bierschenk T., Blundo G., Jaffré Y. \& M. Tidjani Alou (eds), Une anthropologie entre rigueur et engagement. Essais autour de l'œuvre de Jean-Pierre Olivier de Sardan. Leiden, Paris : APAD-Karthala: 163-186.

Lewis, D. 2009. « Anthropology, Development and the 'Perpetual Present'. Knowledge, Power and Practice ». In Hagberg S. and C. Widmark (eds). The ethnographic practice and public aid. Methods and meanings in development cooperation. Uppsala Studies in Cultural Anthropology, 45 : 53-71.

Mbetoumou M., Joiris D.V., Abega S.C. 2010. « La gestion par le haut de la redevance forestière annuelle communautaire.» in Joiris D.V. \& P. Bigombe Logo (eds), La gestion participative des forêts d'Afrique centrale. Un modèle à l'épreuve de la réalité. Versailles : Editions QUAE, Collection Synthèses.

Mosse, D., Farrington J. \& A. Rew (eds). 1998. Development as process. Concepts and methods for working with complexity. London and New York: Routledge Research.

Mosse, D. 2004. « Is good policy unimplementable? Reflections on the ethnography of aid policy and practice», Development change 35 (4): 639-671.

Muller, P. 2008. Les politiques publiques, Paris: PUF (Coll. Que sais-je?). 128 p.

Nguimbi, L., Roulet, P.-A., \& Nzang Oyono, C. 2010. « Une gestion locale assumée par l'opérateur privé : le cas de la CEB au Gabon.», in Joiris D.V. et P. Bigombe Logo (eds). La gestion participative des forêts d'Afrique centrale. Un modèle à l'épreuve de la réalité. Versailles : Editions QUAE, Collection Synthèses.

Olivier de Sardan, J.-P. 2004. « Le chaînon manquant », Courrier de la planète, 74 : 36-40.

-. 1995. Anthropologie et développement. Essai en socio-anthropologie du changement social, Paris : APAD-Karthala.

Picouet, M., Boissau S., Brun B., Rossi G., Sghaier M Et J. Weber 2004. «Le renouvellement des théories population-environnement », in Picouet M., Sghaier M., Genin D., Abaab A., Guillaume H. et M. Elloumi (eds). Environnement et sociétés rurales en mutation. Approches alternatives, Paris, editions IRD: $17-43$.

Quarles Van Ufford, P., Dirk Kruyt P. \& Downing T. 1988. The hidden crisis in development: development bureaucracies. Amsterdam, Free University Press.

Schwartz, N. B. 1981. "Anthropological views of community and community development ", Human Organization, 40 (4): 313-322.

Solly, H. 2010. « Donner, recevoir ou prendre ? La dynamique de la 'participation' dans la réserve du Dja (Cameroun) ", in Joiris D.V. et P. Bigombe Logo (eds). La gestion participative des forêts d'Afrique centrale. Un modèle à l'épreuve de la réalité. Versailles : Editions QUAE, Collection Synthèses.

Windey, C. 2008-2009. Lutte contre la pauvreté et politiques participatives : aspects anthropologiques de la décentralisation du secteur forestier camerounais. Mémoire de Master en Anthropologie à finalité approfondie, Faculté des Sciences Sociales, Politiques et Economiques, Université Libre de Bruxelles. 


\section{NOTES}

1. La définition des politiques participatives qui sont multiformes dans le Bassin congolais s'avère épineuse. Pour la circonscription de l'objet de recherche, nous renvoyons à Joiris \& Bigombe Logo (2010).

2. Programme collectif de recherche GEPAC - UE 2003-2008 - voir Joiris et Bigombe, 2008 ; Joiris et Bigombe, 2010; particulièrement, dans cette dernière publication, les études de cas de Nguimbi et al. et Mbetoumou et al. sur les retombées locales de la fiscalité décentralisée dans le domaine de l'exploitation industrielle du bois ; Bigombe et al. sur les forêts communautaires ; Bigombe et Roulet sur les zones de chasse à vocation communautaire ; Solly, sur les retombées de l'approche participative dans les aires protégées; ainsi que Windey, 2009 sur la RFA et les forêts communautaires.

3. Les comités laissent transparaitre une déconnection par rapport aux réseaux associatifs préexistants et un trop faible suivi de l'évolution des enjeux de représentativité qui se jouent en leur sein (Binot \& Joiris, 2007 ; Binot et. al. 2007 ; Binot et al. 2009 ; Binot et al. 2010; Arnoldussen et al. 2008).

\section{RÉSUMÉS}

La littérature grise en sciences sociales, lorsqu'elle est produite dans le contexte des projets, comporte des particularités cognitives spécifiques qui entravent une contribution opératoire aux politiques de coopération au développement. Dans ce cas, ce n'est pas la littérature en anthropologie du développement qui est mobilisée pour la constitution de la boîte à outils de la recherche appliquée. Il s'agit d'un autre cadre cognitif a-historique, sectoriel et non cumulatif dont les particularités heuristiques sont notamment l'incomparabilité et la non prédictibilité. Ceci mène à la récurrence d'un problème dans la pratique appliquée consistant à entretenir une confusion entre recommandations structurelles et conjoncturelles. Cette contribution est illustrée par des exemples tirés d'une étude pluridisciplinaire sur la gestion participative des forêts dans le Bassin congolais

The grey litterature in social sciences, especially when it is produced in development projects, consists of specific cognitive particularities that prevent an operational contribution to development policies. In this case it is not the anthropology of development literature that is used to construct a toolbox for applied research. Instead it is an a-historical, sectorial and nonaccumulative cognitive framework with its heuristic particularities, such as non-comparability and non-predictability. This leads to a recurrent problem in applied practice that sustains a confusion of structural and conjunctural recommendations. This article contributes with examples from a multidisciplinary study of participatory forest management in the Congo Bassin. 